\title{
EXTREME MEASURABLE SELECTIONS
}

\author{
JERRY A. JOHNSON ${ }^{1}$
}

\begin{abstract}
The extreme points of the set of measurable selections for a set-valued mapping are characterized. As a corollary, the extreme points of the unit ball of the space of "vectorvalued $L^{p}$ functions"' are characterized, thus generalizing results of Sundaresan.
\end{abstract}

1. Introduction. Let $E$ be a separable Banach space and $(S, \mathscr{A}, \mu)$ a measure space. A function $f: S \rightarrow E$ is called measurable if $f^{-1}(B) \in \mathscr{A}$ for each Borel subset $B$ of $E$. For $1 \leqq p<\infty, L_{p}=L_{p}(S, \mathscr{A}, \mu ; E)$ denotes the Banach space of measurable functions $f: S \rightarrow E$ such that

$$
\|f\|_{p}=\left[\int\|f(s)\|^{p} d \mu(s)\right]^{1 / p}<\infty .
$$

We will always identify functions that are equal almost everywhere.

In [11] Sundaresan shows that (with a suitable change in our definition, even for nonseparable $E$ ) if $\|f\|_{p}=1$ and $f(s) /\|f(s)\| \in$ ext $U$ for almost all $s \in S$, then $f$ is an extreme point of the unit ball of $L_{p}$ where $1<p<\infty$, $S$ is a locally compact Hausdorff space, $\mu$ is a regular Borel measure, and ext $U$ is the set of extreme points of the unit ball $U$ of $E$. In the case where $E$ is a separable conjugate space, Theorem 2 in [11] establishes the converse and gives a characterization of the extreme points of the set of measurable functions $f: S \rightarrow U$. These results generalize those of [5]. Other earlier work for $S=[0,1]$ and $E$ finite dimensional was done by Karlin [8] and Aumann [1]. (When the author originally submitted this note, he was unaware of references [10] and [11]. He thanks the referee for calling them to his attention.)

In Proposition 1 of this note we give a characterization of the extreme points of the set of measurable selections for a set-valued function $F$. (It was suggested by Aumann in [1, p. 11] that this could be done if $E$ were finite dimensional and $F$ had compact convex values.) From this, we obtain four corollaries. Corollaries 2 and 4 strengthen [11, Theorem 2]

\footnotetext{
Received by the editors August 1, 1972 and, in revised form, March 29, 1973.

AMS (MOS) subject classifications (1970). Primary 28A05, 46E40.

${ }^{1}$ Supported in part by a grant from the Oklahoma State University College of Arts and Sciences Office of Research and Graduate Studies.
}

(c) American Mathematical Society 1974 
and [1, Proposition 6.1]. Considerations of this sort arise not only in the applications mentioned in [1] but also in control theory (see [9]). Corollary 5 provides a generalization of [11, Theorem 2] to the case where $(S, \mathscr{A}, \mu)$ is a complete measure space and $E$ is a separable Banach space.

2. The characterizations. Let $M$ and $N$ be separable metric spaces and $\mu$ a Borel measure on $M$. By a $\mu$-measurable subset of $M$, we mean in the usual Carathéodory sense (see [2], e.g.). A function $f: M \rightarrow N$ is called $\mu$-measurable [resp. Borel measurable] if $f^{-1}(B)$ is $\mu$-measurable [resp. a Borel set] for each Borel set $B \subset N$.

A subset of a metric space is called analytic (or Souslin) if it is the continuous image of a Borel set in some complete separable metric space. (See [3] and [4] concerning analytic sets.)

The following theorem was proved by von Neumann [6, Lemma 5, p. 448] with $N$ taken as the reals. A careful examination of the proof reveals that it is valid in the more general setting stated below.

Theorem 1 (von NeumanN). Let $M$ and $N$ be complete separable metric spaces, $A$ an analytic subset of $M$, and $g: A \rightarrow N$ continuous. Let $\mu$ be a Borel measure on $N$. Then $g(A)$ is $\mu$-measurable and there exists a $\mu$-measurable mapping $\phi: g(A) \rightarrow M$ such that $g(\phi(x))=x$ for each $x \in g(A)$.

Following [1] we let $2^{S}$ denote the subsets of $S$. The graph of a mapping $F: T \rightarrow 2^{S}$ is denoted by $\mathscr{G}_{F}$ and is defined to be $\{(t, s) \mid s \in F(t)\} . F$ is called Borel measurable or analytic according as its graph is. We note, as pointed out in $[\mathbf{1}$, p. 2], that a point-valued function is Borel measurable if and only if its graph is. In [1, Proposition 2.1] Aumann observed (for $S=[0,1]$ and $\mu$ a Lebesgue measure) the following consequence of Theorem 1 above.

Corollary 1. Let $S_{1}$ and $S_{2}$ be complete separable metric spaces, $F: S_{1} \rightarrow 2^{S_{2}}$ analytic, $F(s) \neq \varnothing$ for each $s$, and $\mu$ a Borel measure on $S_{1}$. Then there is a $\mu$-measurable function $f: S_{1} \rightarrow S_{2}$ with $f(s) \in F(s)$ for each $s \in S$.

Proof. In Theorem 1 above take $M=S_{1} \times S_{2}, N=S_{1}, A=\mathscr{G}_{F}$ and $g\left(s_{1}, s_{2}\right)=s_{1}$. The required selection $f$ is the second component of $\phi$.

Throughout the remainder of this paper, unless otherwise explicitly stated, $E$ will be a separable Banach space, $S$ a separable complete metric space, and $\mu$ a (positive) Borel measure on $S$.

We denote the complement of a set $A$ by $\tilde{A}$, and $B \sim A$ means $B \cap \tilde{A}$. $B-A$ and $B+A$ are used, for subsets of $E$, to mean $\{x-y \mid x \in B, y \in A\}$ and $\{x+y \mid x \in B, y \in A\}$ respectively. 
Lemma 1. Let $F$ and $G$ be Borel measurable from $S$ into $2^{E}$. The mappings $H_{i}, 1 \leqq i \leqq 5$, defined below are Borel measurable.

(1) $H_{1}: s \rightarrow F(s) \cap G(s)$.

(2) If $A \subset S$ is a Borel set, $H_{2}(s)=F(s)$ for $s \in A$ and $H_{2}(s)=G(s)$ for $s \in \tilde{A}$.

(3) If $f: S \rightarrow E$ is Borel measurable and $\lambda$ is a scalar, $H_{3}: s \rightarrow f(s)+\lambda F(s)$.

(4) If $B \subset E$ is a Borel set, $H_{4}: s \rightarrow B$ for all $s \in S$.

(5) $H_{5}(s)=F(s) \times G(s)$.

Proof. (1) $\mathscr{G}_{H} \cap \mathscr{G}_{G_{i}}=\mathscr{G}_{H_{1}}$

(2) $\mathscr{G}_{H_{2}}=\left[\mathscr{G}_{F} \cap(A \times E)\right] \cup\left[\mathscr{G}_{G} \cap(\tilde{A} \times E)\right]$.

(3) Let $\phi: S \times E \rightarrow S \times E$ be defined by $\phi(s, x)=\left(s, \lambda^{-1}(x-f(s))\right)$ if $\lambda \neq 0$. Then $\phi$ is Borel measurable and $\phi^{-1} \mathscr{G}_{F^{\prime}}=\mathscr{G}_{H_{3}}$. (The case $\lambda=0$ is trivial.)

(4) $\mathscr{G}_{H_{4}}=S \times B$.

(5) $\mathscr{G}_{H_{5}}=\phi^{-1}\left(\mathscr{G}_{F} \times \mathscr{G}_{G}\right)$ where $\phi(s, x, y)=((s, x),(s, y))$.

This completes the proof of Lemma 1.

Proposition 1. Let $F: S \rightarrow 2^{E}$ be Borel measurable with $F(s)$ convex and nonempty for each $s \in S$. Let $F_{1}(s)=F(s) \sim \operatorname{ext} F(s)$ and suppose that $\left\{s \mid F_{1}(s) \neq \varnothing\right\}$ is a Borel set. $\mathscr{S}_{F}$ denotes the set of $\mu$-measurable functions $f: S \rightarrow E$ such that $f(s) \in F(s)$ for almost all $s \in S$. Then $f \in \operatorname{ext} \mathscr{S}_{F}$ if and only if $f(s) \in \operatorname{ext} F(s)$ for almost all $s \in S$.

Proof. That the condition is sufficient for $f$ to be in ext $\mathscr{S}_{F}$ is clear.

Let $A=\left\{s \mid f(s) \in F_{1}(s)\right\}$ and suppose $A$ is not of $\mu$-measure zero. Define $F_{2}(s)=(F(s) \times F(s)) \cap(E \times E \sim \Delta)$, where $\Delta$ is the diagonal of $E \times E$. $\mathscr{G}_{F_{2}}$ is a Borel set by Lemma 1 , and the mapping $(s, x, y) \rightarrow(s, 1 / 2(x+y))$ sends $\mathscr{G}_{F_{2}}$ continuously onto $\mathscr{G}_{F_{1}}$. Hence $\mathscr{G}_{F_{1}}$ is analytic. From [2, Propositions 13, 14, p. 97] it follows that there is a Borel measurable function $g: S \rightarrow E$ such that $g=f$ a.e. Let $B=\left\{s \mid g(s) \in F_{1}(s)\right\}$. Now, $\mathscr{G}_{g} \cap \mathscr{G}_{F_{1}}$ is analytic since each graph is (see [3, p. 454 and p. 482]). If $\pi_{1}$ is the canonical projection of $S \times E$ on $S$, then $\pi_{1}\left(\mathscr{G}_{g} \cap \mathscr{G}_{F_{1}}\right)=B$, and therefore $B$ is analytic. $S$ complete and separable implies that $B$ is $\mu$-measurable (see [4, Theorem 5.5, p. 50 and Theorem 7.4, p. 52]). Since $g=f$ a.e., the symmetric difference of $A$ and $B$ is of measure zero. It follows that $\mu B>0$ since otherwise $\mu A=0$, a contradiction. Now, $\mu$ is regular (see [2, Corollary 2, p. 347]) so there is a compact set $K \subset B$ with $\mu K>0$. Let

$$
\begin{aligned}
G(s) & =[(g(s)-F(s)) \cap(-g(s)+F(s))] \sim\{0\}, & & \text { if } s \in K, \\
& =\{0\}, & & \text { if } s \notin K .
\end{aligned}
$$

By Lemma $1, G$ is Borel measurable and, since $G(s) \neq \varnothing$ for each $s$, 
we may apply Corollary 1 to obtain a $\mu$-measurable function $h: S \rightarrow E$ such that $h \in \mathscr{S}_{G}$. This says that $h$ does not vanish on $K$ and that $g+h$ and $g-h$ belong to $\mathscr{S}_{F}$. Since $g=f$ a.e., it follows that $f$ is not an extreme point of $\mathscr{S}_{F}$. This completes the proof of the proposition.

The following corollary extends the $L^{\infty}$ case of Theorem 2 in [11].

Corollary 2. If $K$ is a nonempty, convex, Borel subset of $E$ and $\dot{\mathscr{S}}_{K}$ is the set of $\mu$-measurable functions $f: S \rightarrow K$, then $f \in \operatorname{ext} \mathscr{S}_{K}$ if and only if $f(s) \in \operatorname{ext} K$ for almost all $s \in S$.

The next corollary has two consequences, the second of which contains a converse of Theorem 2 in [5].

Corollary 3. Let $K$ be a closed, convex, nonempty subset of $E$ and $\mu$ a Borel measure on $K$. If $\mu(K \sim$ ext $K)>0$, there is a Borel measurable function $g: K \rightarrow E$ such that $g \neq 0$ on a set of positive measure and such that $x \pm g(x) \in K$ for almost all $x \in K$.

Proof. First note that $K \sim \operatorname{ext} K$ is analytic (see the proof of Proposition 1) and hence is $\mu$-measurable. Now, letting $S=K$ in Corollary 2, we see that the identity map on $K$ is not an extreme point of $\mathscr{S}_{K}$. Thus, there is a $\mu$-measurable function $g_{0}: K \rightarrow E$ such that $x \pm g_{0}(x) \in K$ for almost all $x \in K$ and such that $g_{0} \neq 0$ on a set of positive measure. Let $g$ be a Borel measurable function equal $\mu$-a.e. to $g_{0}$.

Corollary 4. Let $K$ be a nonempty, closed, convex subset of $E$ and $(S, \mathscr{A}, v)$ a measure space complete in the measure theoretic sense. Let $\mathscr{S}$ be the set of functions $f: S \rightarrow K$ that are $\mathscr{A}$-measurable; i.e., $f^{-1}(B) \in \mathscr{A}$ for each Borel set $B \subset K$. (We continue to identify functions equal v-a.e.) Then $f \in \operatorname{ext} \mathscr{S}$ if and only if $f(s) \in$ ext $K$ for $v$-almost all $s \in S$.

Proof. Define $\mu B=v f^{-1}(B)$ for each Borel set $B \subset K$, and suppose that $A=\{s \mid f(s) \notin$ ext $K\}$ is not of $\nu$-measure zero. $K \sim$ ext $K$ is analytic and therefore $\mu$-measurable. If $\mu(K \sim$ ext $K)=0$, then by the regularity of $\mu$, there is a Borel set $B \supset K \sim$ ext $K$ with $\mu B=0$. Thus, $f^{-1}(B) \supset A$ and $v f^{-1}(B)=0$. By the completeness of $(S, \mathscr{A}, v)$, we have $A \in \mathscr{A}$ and $\nu A=0$, a contradiction. Hence, $\mu(K \sim \operatorname{ext} K)>0$ and we choose $g$ to be the Borel measurable function guaranteed by Corollary 3. Then $g \circ f \neq 0$ on a set of positive $v$-measure and $f(s) \pm g(f(s)) \in K$ for $v$-almost all $s \in S$. Hence $f \notin$ ext $\mathscr{S}$. The converse is clear.

Corollary 5. Let $(S, \mathscr{A}, v)$ be as in Corollary 4. Then $f$ is an extreme point of the unit ball of $L_{p}=L_{p}(S, \mathscr{A}, v ; E), 1<p<\infty$, if and only if $\|f\|_{n}=1$, and for almost all $s$ in the support $S_{f}$ of $f, f(s) /\|f(s)\|$ is an extreme point of the unit ball $U$ of $E$. 
Proof. Let $f$ be an extreme point of the unit ball of $L_{p}$. We apply Corollary 4 to the measure space $\left(S_{f}, \mathscr{A}, v\right)$, the convex set $U$, and the function $h(s)=f(s) /\|f(s)\|$ on $S_{f}$. If $h$ is not an extreme point of $\mathscr{S}$, then there exist $h_{1}, h_{2} \in \mathscr{S}$ such that $h_{1} \neq h_{2}$ on a set of positive measure and $h=1 / 2\left(h_{1}+h_{2}\right)$. Let $f_{j}(s)=\|f(s)\| h_{j}(s)$ for $s \in S_{f}$ and $f_{j}(s)=0$ for $s \in S \sim S_{f}$. It then follows that $f=1 / 2\left(f_{1}+f_{2}\right)$, each $f_{j}$ is in the unit ball of $L_{p}$, and $f_{1} \neq f_{2}$ on a set of positive measure. This is a contradiction. Thus, $h$ is an extreme point of $\mathscr{S}$, so by Corollary 4 , we have $h(s) \in \operatorname{ext} U$ for almost all $s \in S$.

The proof of the converse may be taken verbatim from [5].

3. Closing remarks. The hypothesis of separability and completeness of $E$ and $S$ is necessary to determine that an analytic set is $\mu$-measurable, and that a Borel set in $S \times E$ is in the product sigma-algebra.

If $K$ is a compact convex subset of $E$ then ext $K$ is a $\mathscr{G}_{\delta}$ set (see [7, Proposition 1.3]). The author does not know whether ext $U$ is a Borel set for arbitrary separable $E$. As noted earlier, $U \sim \operatorname{ext} U$ is analytic. By [3, Corollary 1, p. 486], therefore, to prove ext $U$ is a Borel set it is enough to prove that it is analytic.

ACKNOWLEDGMENT. The author takes great pleasure in expressing his gratitude to Robert $F$. Kaufman for contributing many enlightening comments and helpful suggestions.

\section{REFERENCES}

1. R. J. Aumann, Integrals of set-valued functions, J. Math. Anal. Appl. 12 (1965), 1-12. MR 32 \#2543.

2. N. Dinculeanu, Vector measures, Internat. Series of Monographs in Pure and Appl. Math., vol. 95, Pergamon Press, Oxford; VEB Deutscher Verlag der Wissenschaften, Berlin, 1967. MR 34 \#6011b.

3. K. Kuratowski, Topologie. Vol. I, 2nd ed., Monografie Mat., Tom 20, WarszawaWroclaw, 1948; English transl., New ed., rev. and aug., Academic Press, New York; PWN, Warsaw, 1966. MR 10, 389; 36 \#840.

4. S. Saks, Theory of the integral, reprint, Dover, New York, 1964. MR 29 \#4850.

5. K. Sundaresan, Extreme points of the unit cell in Lebesgue-Bochner function spaces. I, Proc. Amer. Math. Soc. 23 (1969), 179-184. MR 40 \#719.

6. J. von Neumann, On rings of operators. Reduction theory, Ann. of Math. (2) 50 (1949), 401-485. MR 10, 548.

7. R. R. Phelps, Lectures on Choquet's theorem, Van Nostrand, Princeton, N.J., 1966. MR 33 \#1690.

8. S. Karlin, Extreme points of vector functions, Proc. Amer. Math. Soc. 4 (1953), 603-610. MR 15, 109.

9. H. Hermes and J. LaSalle, Functional analysis and time optimal control, Academic Press, New York, 1969. 
10. K. Sundaresan, Extreme points of convex sets and selection theorems, Set-Valued Mappings, Selections and Topological Properties of $2^{x}$ (Proc. Conf., SUNY, Buffalo, N.Y., 1968), Lecture Notes in Math., vol. 171, Springer, Berlin, 1970, pp. 82-87. MR 45 \#1106.

11. - Extreme points of the unit cell in Lebesgue-Bochner function spaces, Colloq. Math. 22 (1970), 111-119. MR 43 \#2493.

Department of Mathematics and Statistics, Oklahoma State University, STILlWATER, OKLAHOMA 74074 